\title{
Trajectory Estimation of Aircraft in a Double-Satellite Passive Positioning System with the Adjoint Method
}

\author{
Anzhou Cao, ${ }^{1}$ Yanqiu Gao, ${ }^{1}$ Jicai Zhang, ${ }^{2}$ and Xianqing $\mathrm{Lv}^{1}$ \\ ${ }^{1}$ Laboratory of Physical Oceanography, Ocean University of China, Qingdao 266003, China \\ ${ }^{2}$ Laboratory of Coast and Island Development, Nanjing University, Nanjing 210093, China \\ Correspondence should be addressed to Jicai Zhang; jicai_zhang@163.com
}

Received 7 March 2013; Accepted 15 May 2013

Academic Editor: Antonio F. Bertachini A. Prado

Copyright (c) 2013 Anzhou Cao et al. This is an open access article distributed under the Creative Commons Attribution License, which permits unrestricted use, distribution, and reproduction in any medium, provided the original work is properly cited.

\begin{abstract}
A double-satellite passive positioning system is constructed based on the theory of space geometry, where two observation coordinate systems and a fundamental coordinate system exist. In each observation coordinate system, there exists a ray from the observation satellite to the aircraft. One difficulty lies in that these two rays may not intersect due to the existence of various errors. Under this situation, this work assumes that the middle point of common perpendicular between two rays is the actual position of aircraft. Based on the theory of space geometry, the coordinates of aircraft in the fundamental coordinate system can be determined. A dynamic model with the adjoint method is developed to estimate the trajectory of aircraft during the process of rocket propulsion. By assimilating observations, the trajectory of aircraft can be calculated. Numerical experiments are designed to validate the reasonability and feasibility of this model. Simulated results indicate that even by assimilating a small number of observations, the trajectory of aircraft can be estimated. In addition, the trajectory estimation can become more accurate when more observations are assimilated to the model.
\end{abstract}

\section{Introduction}

The determination of the position of an aircraft by a passive observer is a typical navigation problem [1]. In this paper, a double-satellite passive positioning system is constructed based on the theory of space geometry. In this system, two observation coordinate systems corresponding to two observation satellites and a fundamental coordinate system exist. In each observation coordinate system, there exists a ray from the observation satellite to the aircraft. In the ideal situation where no error exists in the double-satellite passive positioning system, these two rays intersect and the point of intersection is the aircraft. One difficulty lies in that these two rays may not intersect due to the existence of various errors which contain systemic error of observation satellite and random error of measurement. Under this situation, this work assumes that the middle point of common perpendicular between two rays is the actual position of aircraft. Based on the assumption and theory of space geometry, the coordinates of aircraft in the fundamental coordinate system can be determined.
Based on observations of aircraft in the fundamental coordinate system, the trajectory of aircraft could be estimated. Spingarn [1] used an extended Kalman filter to obtain the estimations of optimal filtered position combined with observations. Jamilnia and Naghash [2] used two different approaches, which were separated approach and integrated approach, to optimize trajectories of multistage launch vehicles simultaneously. In this paper, the adjoint method is used to estimate the trajectory of aircraft during the process of rocket propulsion. Based on the theory of inverse problem, the adjoint method is a useful tool for parameter estimation by assimilating observations into numerical models. Lardner [3], Zhang and Lu [4], Guo et al. [5], Cao et al. [6], and Chen et al. [7] studied the inversion of open boundary conditions for ocean dynamic models. As for the inverse problem of other parameters, Lu and Zhang [8] and Zhang et al. [9] inverted spatially varying bottom friction coefficient for the twodimensional tidal model, and Yu and O'Brien [10] estimated wind drag coefficient and eddy viscosity coefficients in an Ekman layer model. Results of their work demonstrate that 
the adjoint method is a useful tool for parameter estimation and can improve the accuracy of simulated results.

This paper is organized as follows. The double-satellite passive positioning system is constructed in Section 2. The dynamic model with the adjoint method is developed to estimate the trajectory of aircraft in Section 3. In Section 4, numerical experiments are designed to validate the reasonability and feasibility of this model. Summaries and conclusions are presented in Section 5.

\section{Double-Satellite Passive Positioning System}

2.1. Transformation of Coordinates. Two coordinate systems, namely, the fundamental and observation coordinate system, are used in a passive positioning system. Figure 1 is a snapshot of these coordinate systems. In the fundamental coordinate system $\left(O_{c}-X_{c} Y_{c} Z_{c}\right)$, the geocenter is taken as the origin. The earth and observation satellite are simplified as two particles and constitute a two-body problem under the effect of gravity. Considering that the time scale of studied problem is much shorter than that of earth rotation, the fundamental coordinate system is regarded as an inertial system. The aircraft is measured by observation satellite in the observation coordinate system $\left(O_{s}-X_{s} Y_{s} Z_{s}\right)$. As shown in the observation coordinate system of Figure 1, the position of observation satellite $\left(O_{s}\right)$ is taken as the origin, $X_{s}$-axis is along the vector $\overrightarrow{\mathrm{O}_{c} \mathrm{O}_{s}}$, and $Z_{s}$-axis is perpendicular to $X_{s}$-axis and pointing to the north exactly. $Y_{s}$-axis is then ascertained following right hand system. Generally speaking, observation satellite would not pass the south and north poles, so the observation coordinate is definite and reasonable.

Considering that observation satellite is passive detective satellite which only measures direction, the aircraft is observed and represented by two dimensionless ratios ( $\alpha$ and $\beta$ ) in the observation coordinate:

$$
\begin{aligned}
& \alpha=\frac{y_{s}}{x_{s}}, \\
& \beta=\frac{z_{s}}{x_{s}},
\end{aligned}
$$

where $x_{s}, y_{s}$, and $z_{s}$ are the coordinates of aircraft in the observation coordinate system. It should be noted that $x_{s}, y_{s}$, and $z_{s}$ are just used to represent the position of aircraft and cannot be measured by one observation satellite.

The key to achieve double-satellite passive position is to transform coordinates from the observation coordinate system to the fundamental one. The basic idea of transformation is described as follows. Assume that $Q$ is an arbitrary point in the observation coordinate system. Three components of vector $\overrightarrow{O_{s} Q}$ along $\overrightarrow{O_{s} X_{s}}, \overrightarrow{O_{s} Y_{s}}$, and $\overrightarrow{O_{s} Z_{s}}$ are set to $\overrightarrow{Q x}, \overrightarrow{Q y}$ and $\overrightarrow{Q z}$, respectively. Considering that $\overrightarrow{O_{s} X_{s}}, \overrightarrow{O_{s} Y_{s}}$, and $\overrightarrow{O_{s} z_{s}}$ are well-determined and can be described by the unit vectors $\vec{i}, \vec{j}$ and $\vec{k}$ in the fundamental coordinate system, $\overrightarrow{Q x}, \overrightarrow{Q y}$, and $\overrightarrow{Q z}$ have the following relation with $\vec{i}, \vec{j}$, and $\vec{k}$ :

$$
\overrightarrow{Q x}=A_{1} \overrightarrow{O_{s} X_{s}}+B_{1} \overrightarrow{O_{s} Y_{s}}+C_{1} \overrightarrow{O_{s} Z_{s}}=a_{1} \vec{i}+b_{1} \vec{j}+c_{1} \vec{k}
$$

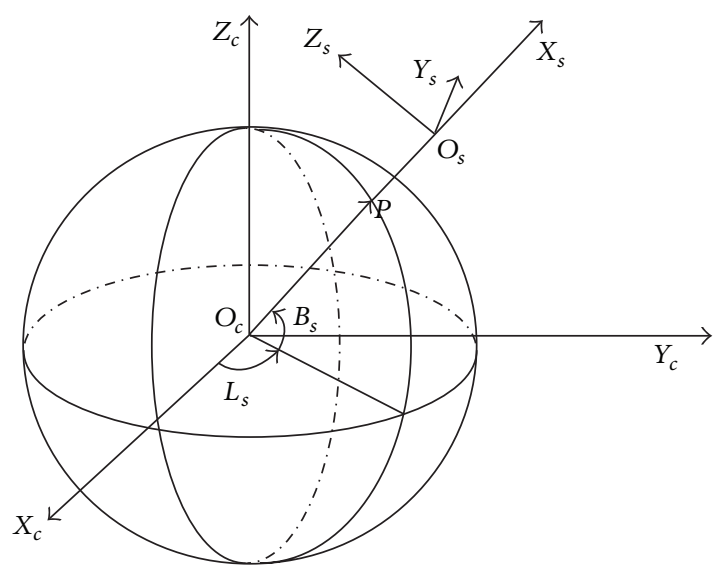

Figure 1: Outline of the fundamental $\left(O_{c}-X_{c} Y_{c} Z_{c}\right)$ and observed $\left(O_{s}-X_{s} Y_{s} Z_{s}\right)$ coordinate system.

$$
\begin{aligned}
& \overrightarrow{Q y}=A_{2} \overrightarrow{O_{s} X_{s}}+B_{2} \overrightarrow{O_{s} Y_{s}}+C_{2} \overrightarrow{O_{s} Z_{s}}=a_{2} \vec{i}+b_{2} \vec{j}+c_{2} \vec{k}, \\
& \overrightarrow{Q z}=A_{3} \overrightarrow{O_{s} X_{s}}+B_{3} \overrightarrow{O_{s} Y_{s}}+C_{3} \overrightarrow{O_{s} Z_{s}}=a_{3} \vec{i}+b_{3} \vec{j}+c_{3} \vec{k},
\end{aligned}
$$

where $A_{1}, A_{2}, A_{3}, B_{1}, B_{2}, B_{3}, C_{1}, C_{2}, C_{3}, a_{1}, a_{2}, a_{3}, b_{1}, b_{2}, b_{3}, c_{1}$, $c_{2}$, and $c_{3}$ are parameters and will be derived in the following part. Then $\overrightarrow{O_{s} Q}$ can be rewritten as

$$
\overrightarrow{O_{s} Q}=\left(a_{1}+a_{2}+a_{3}\right) \vec{i}+\left(b_{1}+b_{2}+b_{3}\right) \vec{j}+\left(c_{1}+c_{2}+c_{3}\right) \vec{k}
$$

which is the description of $Q$ in the fundamental coordinate system. Detailed derivations are displayed as follows.

Assume that $O_{s}$ is $\left(x_{0}, y_{0}, z_{0}\right)$ in the fundamental coordinate system, $Q$ is $\left(x_{1}, y_{1}, z_{1}\right)$ in the observation coordinate system, and $\left(x_{2}, y_{2}, z_{2}\right)$ in the fundamental one. Because $\overrightarrow{O_{s} X_{s}}$ is along vector $\overrightarrow{O_{c} O_{s}}$, the unit vector of $\overrightarrow{O_{s} X_{s}}$ in the fundamental coordinate system is

$$
\overrightarrow{v_{1}}=\left(\frac{x_{0}}{\sqrt{x_{0}^{2}+y_{0}^{2}+z_{0}^{2}}}, \frac{y_{0}}{\sqrt{x_{0}^{2}+y_{0}^{2}+z_{0}^{2}}}, \frac{z_{0}}{\sqrt{x_{0}^{2}+y_{0}^{2}+z_{0}^{2}}}\right) .
$$

Considering that $\overrightarrow{O_{s} Z_{s}}$ points to the north exactly, $\overrightarrow{O_{s} Z_{s}}$ lies in the plane $O_{s} O_{c} Z_{c}$. As a result, direction vector of ${\overrightarrow{O_{s} Y_{s}}}_{\text {is }}$ also the normal vector of $O_{s} O_{c} Z_{c}$. Then unit vector of $\overrightarrow{O_{s} Y_{s}}$ in the fundamental coordinate system can be described as

$$
\overrightarrow{v_{2}}=\left(\frac{-y_{0}}{\sqrt{x_{0}^{2}+y_{0}^{2}}}, \frac{x_{0}}{\sqrt{x_{0}^{2}+y_{0}^{2}}}, 0\right) \text {. }
$$


As unit vectors of $\overrightarrow{O_{s} X_{s}}$ and $\overrightarrow{O_{s} Y_{s}}$ have been determined, that of $\overrightarrow{\mathrm{O}_{s} z_{s}}$ can be calculated by vector multiplication cross

$$
\begin{gathered}
\overrightarrow{v_{3}}=\left(\frac{-x_{0} z_{0}}{\sqrt{x_{0}^{2} z_{0}^{2}+y_{0}^{2} z_{0}^{2}+\left(x_{0}^{2}+y_{0}^{2}\right)^{2}}},\right. \\
\frac{-y_{0} z_{0}}{\sqrt{x_{0}^{2} z_{0}^{2}+y_{0}^{2} z_{0}^{2}+\left(x_{0}^{2}+y_{0}^{2}\right)^{2}}} \\
\left.\frac{x_{0}^{2}+y_{0}^{2}}{\sqrt{x_{0}^{2} z_{0}^{2}+y_{0}^{2} z_{0}^{2}+\left(x_{0}^{2}+y_{0}^{2}\right)^{2}}}\right) .
\end{gathered}
$$

Consequently, $Q$ in the fundamental coordinate system is described as

$$
\left(x_{2}, y_{2}, z_{2}\right)=x_{1} \overrightarrow{v_{1}}+y_{1} \overrightarrow{v_{2}}+z_{1} \overrightarrow{v_{3}}+\left(x_{0}, y_{0}, z_{0}\right)
$$

2.2. Double-Satellite Passive Positioning System. As described before, observation satellite is passive detective satellite and can measure direction only. It means that the position of aircraft can be determined only when two or more observation satellites are available. In this paper, a double-satellite passive positioning system is constructed to study the positioning problem. This means that there exist two observation coordinate systems, of which the origins are the two observation satellites, respectively. In each observation coordinate system, there exists a ray from the observation satellite to the aircraft. In the ideal situation where no error exists in the doublesatellite passive positioning system, these two rays intersect and the intersection point is the aircraft. One difficulty lies in that these two rays may not intersect due to the existence of various errors. Under this situation, this work assumes that the middle point of common perpendicular between two rays is the actual position of aircraft.

As $\alpha, \beta$, and the trajectory information of observation satellites are known, the equation of the ray from the observation satellite to the aircraft in the fundamental coordinate system can be obtained as long as $(1, \alpha, \beta)$ is transformed from the observation coordinate system to the fundamental one. According to the assumption, the position of aircraft can be determined based on the theory of space geometry. Detailed derivation is displayed as follows. (It should be noted that the unit vector rather than the equation of the ray is used in the derivation.)

Figure 2 is the schematic diagram of the double-satellite passive positioning system. $M$ and $N$ represent two satellites, and $A$ and $B$ represent the observations of the aircraft by satellites $M$ and $N$, respectively. Based on the assumption, the middle point of $A B$ is the actual position of the aircraft, which is represented by $C$ in Figure 2 . In the fundamental coordinate system, assume that the coordinates of $M$ and $N$

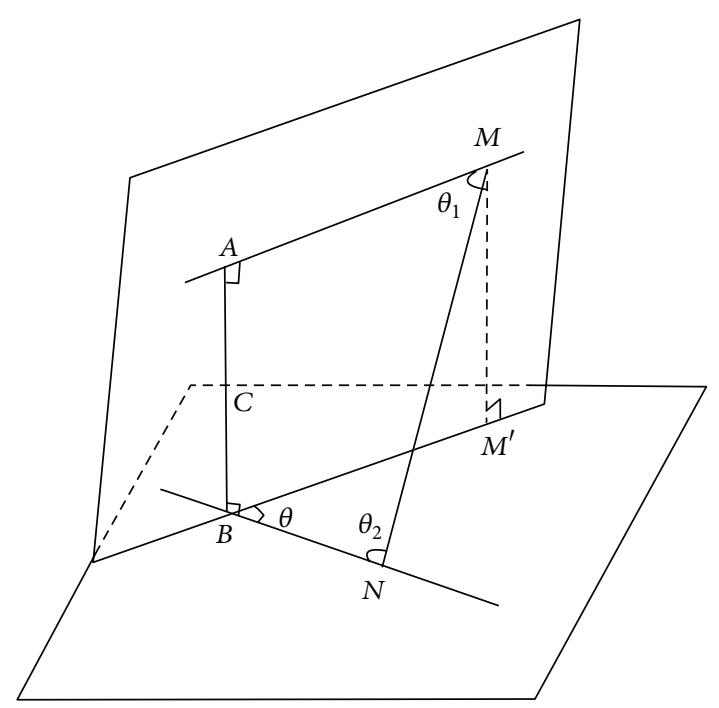

FIGURE 2: Outline of the double-satellite passive positioning system.

are $\left(x_{m}, y_{m}, z_{m}\right)$ and $\left(x_{n}, y_{n}, z_{n}\right)$, and unit vectors of $\overrightarrow{M A}$ and $\overrightarrow{N B}$ are $\left(\varphi_{1}, \varphi_{2}, \varphi_{3}\right)$ and $\left(\phi_{1}, \phi_{2}, \phi_{3}\right)$. Consider

$\theta_{1}$

$=\arccos \left(\frac{\varphi_{1}\left(x_{n}-x_{m}\right)+\varphi_{2}\left(y_{n}-y_{m}\right)+\varphi_{3}\left(z_{n}-z_{m}\right)}{\sqrt{\left(x_{n}-x_{m}\right)^{2}+\left(y_{n}-y_{m}\right)^{2}+\left(z_{n}-z_{m}\right)^{2}}}\right)$,

$\theta_{2}$

$$
\begin{gathered}
=\arccos \left(\frac{\phi_{1}\left(x_{n}-x_{m}\right)+\phi_{2}\left(y_{n}-y_{m}\right)+\phi_{3}\left(z_{n}-z_{m}\right)}{\sqrt{\left(x_{n}-x_{m}\right)^{2}+\left(y_{n}-y_{m}\right)^{2}+\left(z_{n}-z_{m}\right)^{2}}}\right), \\
\theta=\arccos \left(\varphi_{1} \phi_{1}+\varphi_{2} \phi_{2}+\varphi_{3} \phi_{3}\right) .
\end{gathered}
$$

Then,

$$
\begin{aligned}
|\overrightarrow{M A}|= & \frac{\cos \theta_{1}+\cos \theta_{2} \cdot \cos \theta}{\sin ^{2} \theta} \\
& \cdot \sqrt{\left(x_{n}-x_{m}\right)^{2}+\left(y_{n}-y_{m}\right)^{2}+\left(z_{n}-z_{m}\right)^{2}}, \\
|\overrightarrow{N B}|= & \frac{\cos \theta_{2}+\cos \theta_{1} \cdot \cos \theta}{\sin ^{2} \theta} \\
& \cdot \sqrt{\left(x_{n}-x_{m}\right)^{2}+\left(y_{n}-y_{m}\right)^{2}+\left(z_{n}-z_{m}\right)^{2}} .
\end{aligned}
$$

Finally, the coordinate of aircraft $(C)$ in the fundamental coordinate system can be calculated as

$$
\begin{gathered}
\left(x_{a}, y_{a}, z_{a}\right)=\left(x_{m}, y_{m}, z_{m}\right)+\overrightarrow{\mid M A} \mid\left(\varphi_{1}, \varphi_{2}, \varphi_{3}\right), \\
\left(x_{b}, y_{b}, z_{b}\right)=\left(x_{n}, y_{n}, z_{n}\right)+\overrightarrow{|N B|}\left(\phi_{1}, \phi_{2}, \phi_{3}\right),
\end{gathered}
$$




$$
\begin{aligned}
\left(x_{c}, y_{c}, z_{c}\right)=\frac{1}{2}[ & \left(x_{m}, y_{m}, z_{m}\right)+\left(x_{n}, y_{n}, z_{n}\right) \\
& \left.+|\overrightarrow{M A}|\left(\varphi_{1}, \varphi_{2}, \varphi_{3}\right)+|\overrightarrow{N B}|\left(\phi_{1}, \phi_{2}, \phi_{3}\right)\right] .
\end{aligned}
$$

\section{Dynamic model}

As mentioned in Section 2, the coordinates of aircraft in the fundamental coordinate system are determined by the double-satellite passive positioning system, and they will be used as observations in the dynamic model. If adequate observations are obtained, the trajectory of the aircraft can be estimated by using the dynamic model with the adjoint method.

3.1. Forward Model (Governing Equation). The governing equation of the aircraft during the process of rocket propulsion in the fundamental coordinate system can be described as follows:

$$
\ddot{\vec{r}}(t)=\vec{F}_{e}+\vec{F}_{T},
$$

where $\vec{r}(t)$ is the vector of position, $t$ is time, $\ddot{\vec{r}}(t)$ is the double derivative in time of $\vec{r}(t)$, that is, acceleration, $G_{m}$ is the constant of earth attraction, $\vec{F}_{e}=-\left(G_{m} /|\vec{r}(t)|^{3}\right) \vec{r}(t)$ is the acceleration caused by universal gravitation, and $\vec{F}_{T}$ is the acceleration caused by rocket propulsion, which is taken as the unknown parameter and will be optimized by the adjoint method. Assume that $F_{x}, F_{y}$ and $F_{z}$, are the $x$-, $y$-, and $z$ components of $\vec{F}_{T}$, respectively, (11) can be changed into

$$
\begin{aligned}
& \frac{d^{2} x}{d t^{2}}=-\frac{G_{m}}{r^{3}} x+F_{x}, \\
& \frac{d^{2} y}{d t^{2}}=-\frac{G_{m}}{r^{3}} y+F_{y}, \\
& \frac{d^{2} z}{d t^{2}}=-\frac{G_{m}}{r^{3}} z+F_{z} \\
& r=\sqrt{x^{2}+y^{2}+z^{2}}
\end{aligned}
$$

where $x, y$, and $z$ are three components of $\vec{r}(t)$.

3.2. Adjoint Model and Corrections. The cost function is defined as

$$
J=\frac{\kappa}{2} \int_{t_{1}}^{t_{2}}\left[(x-\widehat{x})^{2}+(y-\widehat{y})^{2}+(z-\widehat{z})^{2}\right] d t,
$$

where $\kappa$ is the unit matrix [8] $\widehat{x}, \widehat{y}, \widehat{z}, x, x$, and $z$ are observations and simulated coordinate of the aircraft, respectively. Then the Lagrangian function can be expressed as

$$
L=J+\int_{t_{1}}^{t_{2}}\left[\lambda\left(\frac{d^{2} x}{d t^{2}}+\frac{G_{m}}{r^{3}} x-F_{x}\right)\right.
$$

$$
\begin{aligned}
& +\mu\left(\frac{d^{2} y}{d t^{2}}+\frac{G_{m}}{r^{3}} y-F_{y}\right) \\
& \left.+\nu\left(\frac{d^{2} z}{d t^{2}}+\frac{G_{m}}{r^{3}} z-F_{z}\right)\right] d t,
\end{aligned}
$$

where $\lambda, \mu$, and $\nu$ are the adjoint variables (namely, Lagrangian multipliers) of $x, y$, and $z$, respectively.

According to the theory of Lagrangian multiplier method, we obtain the first-order derivatives of Lagrangian function with respect to all the variables,

$$
\begin{aligned}
\frac{\partial L}{\partial \lambda}=0, & \frac{\partial L}{\partial \mu}=0, & \frac{\partial L}{\partial \nu}=0, \\
\frac{\partial L}{\partial x}=0, & \frac{\partial L}{\partial y}=0, & \frac{\partial L}{\partial z}=0, \\
\frac{\partial L}{\partial F_{x}}=0, & \frac{\partial L}{\partial F_{y}}=0, & \frac{\partial L}{\partial F_{z}}=0 .
\end{aligned}
$$

Equation (15) give the forward model described by (12). From (16) the adjoint model can be deduced as

$$
\begin{aligned}
& \frac{d^{2} \lambda}{d t^{2}}+G_{m} \frac{\left(y^{2}+z^{2}-2 x^{2}\right) \lambda-3 x(y \mu+z \nu)}{r^{5}}+\kappa(x-\widehat{x})=0, \\
& \frac{d^{2} \mu}{d t^{2}}+G_{m} \frac{\left(x^{2}+z^{2}-2 y^{2}\right) \mu-3 y(x \lambda+z \nu)}{r^{5}}+\kappa(y-\widehat{y})=0, \\
& \frac{d^{2} \nu}{d t^{2}}+G_{m} \frac{\left(x^{2}+y^{2}-2 z^{2}\right) \nu-3 z(x \lambda+y \mu)}{r^{5}}+\kappa(z-\widehat{z})=0 .
\end{aligned}
$$

From (17), the corrections of $F_{x}, F_{y}$, and $F_{z}$ are obtained

$$
\begin{aligned}
& F_{x}-\widetilde{F}_{x}=\lambda, \\
& F_{y}-\widetilde{F}_{y}=\mu, \\
& F_{z}-\widetilde{F}_{z}=\nu,
\end{aligned}
$$

where $\widetilde{F}_{x}, \widetilde{F}_{y}, \widetilde{F}_{z}$ and $F_{x}, F_{y}, F_{z}$ are prior and optimized values, receptively. Because the values of cost function decrease along the opposite direction of the gradient, by employing typical steepest descent method [11], $F_{x}, F_{y}$, and $F_{z}$ can be optimized during iterations. 
Equations (12), (18) and (19) are discretized using the finite difference method and are shown as follows:

$$
\begin{aligned}
& \frac{x^{n+1}-2 x^{n}+x^{n-1}}{\Delta t^{2}}=-\frac{G_{m}}{\left(r^{n}\right)^{3}} x^{n}+F_{x}^{n} \text {, } \\
& \frac{y^{n+1}-2 y^{n}+y^{n-1}}{\Delta t^{2}}=-\frac{G_{m}}{\left(r^{n}\right)^{3}} y^{n}+F_{y}^{n} \text {, } \\
& \frac{z^{n+1}-2 z^{n}+z^{n-1}}{\Delta t^{2}}=-\frac{G_{m}}{\left(r^{n}\right)^{3}} z^{n}+F_{z}^{n} \\
& r^{n}=\sqrt{\left(x^{n}\right)^{2}+\left(y^{n}\right)^{2}+\left(z^{n}\right)^{2}}, \\
& \frac{\lambda^{n-1}-2 \lambda^{n}+\lambda^{n+1}}{\Delta t^{2}} \\
& +G_{m} \frac{\left(\left(y^{n}\right)^{2}+\left(z^{n}\right)^{2}-2\left(x^{n}\right)^{2}\right) \lambda^{n}-3 x^{n}\left(y^{n} \mu^{n}+z^{n} v^{n}\right)}{\left(r^{n}\right)^{5}} \\
& +\kappa\left(x^{n}-\widehat{x}^{n}\right)=0, \\
& \frac{\mu^{n-1}-2 \mu^{n}+\mu^{n+1}}{\Delta t^{2}} \\
& +G_{m} \frac{\left(\left(x^{n}\right)^{2}+\left(z^{n}\right)^{2}-2\left(y^{n}\right)^{2}\right) \mu^{n}-3 y^{n}\left(x^{n} \lambda^{n}+z^{n} v^{n}\right)}{\left(r^{n}\right)^{5}} \\
& +\kappa\left(y^{n}-\widehat{y}^{n}\right)=0, \\
& \frac{\nu^{n-1}-2 v^{n}+\nu^{n+1}}{\Delta t^{2}} \\
& +G_{m} \frac{\left(\left(x^{n}\right)^{2}+\left(y^{n}\right)^{2}-2\left(z^{n}\right)^{2}\right) \nu^{n}-3 z^{n}\left(x^{n} \lambda^{n}+y^{n} \mu^{n}\right)}{\left(r^{n}\right)^{5}} \\
& +\kappa\left(z^{n}-\widehat{z}^{n}\right)=0, \\
& F_{x}^{n}-\widetilde{F}_{x}^{n}=\lambda^{n}, \\
& F_{y}^{n}-\widetilde{F}_{y}^{n}=\mu^{n} \text {, } \\
& F_{z}^{n}-\widetilde{F}_{z}^{n}=v^{n} \text {. }
\end{aligned}
$$

The computed process is designed as follows. (a) Run the forward model with prescribed initial values of $F_{x}$, $F_{y}$, and $F_{z}$. (b) Calculate the value of cost function $J$. (c) The difference between simulated results and observations plays as the external driving force of the adjoint model. The adjoint variables can then be obtained through the backward integration of the adjoint model. (d) Based on the corrections and typical steepest descent method, $F_{x}, F_{y}$, and $F_{z}$ can be optimized. Repeating the process aforementioned, the difference between simulated results and observations is decreased. When the convergence criterion is met, the optimization stops.

The convergence criterion could be that the number of iteration steps is prescribed, the last two values of the
TABLE 1: Difference between simulated coordinates and observed ones.

\begin{tabular}{ccccccc}
\hline \multicolumn{8}{c}{ Mean absolute difference (m) } & \multicolumn{4}{c}{ Mean relative difference (\%) } \\
& $x$ & $y$ & $z$ & $x$ & $y$ & $z$ \\
\hline TE1 & 116 & 38 & 71 & 0.0097 & 0.00063 & 0.0057 \\
TE2 & 124 & 40 & 77 & 0.0010 & 0.00065 & 0.0062 \\
TE3 & 160 & 40 & 103 & 0.0013 & 0.00065 & 0.0083 \\
TE4 & 244 & 43 & 162 & 0.0020 & 0.00069 & 0.0130 \\
\hline
\end{tabular}

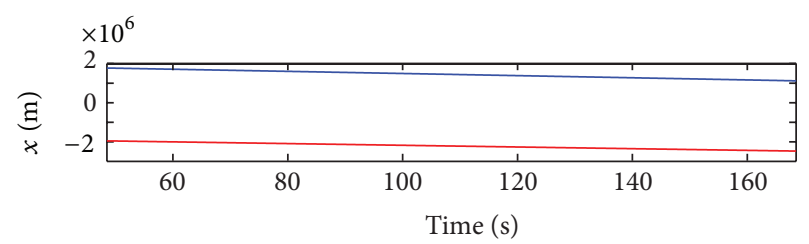

(a)

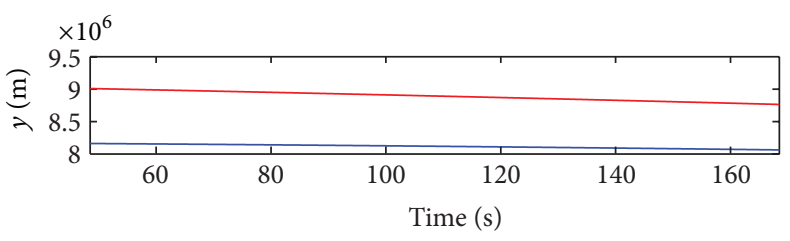

(b)

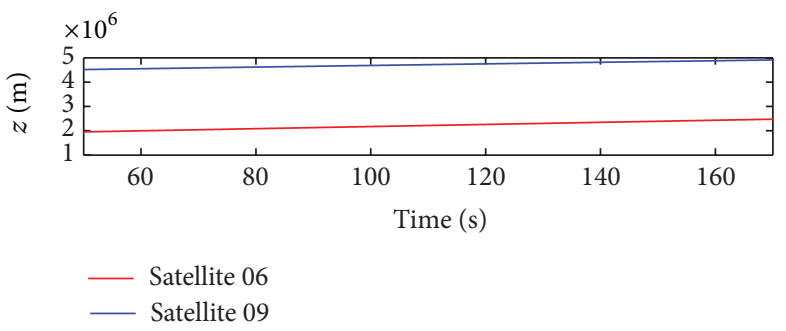

(c)

FIGURE 3: Coordinates of observation satellites in the fundamental coordinate system.

cost function are sufficiently close, the magnitude of the gradient norm is sufficiently small, the discrepancy between the updated and old parameters is sufficiently small, or a combination of these. In this work, the convergence criterion is that the number of iteration steps is equal to 10000 , which leads to satisfied values of cost function and gradient norm and is obtained through a trial process. In addition, this convergence criterion is reasonable and essential to investigate the influence of the number of observations on the accuracy of simulated results in the numerical experiments.

\section{Numerical Experiments}

The data used in the numerical experiments, containing $\alpha, \beta$, and trajectory information of two observation satellites (satellites 06 and 09), are from problem B of the ninth National Graduate Mathematical Contest in Modeling (http://www.njnet.edu.cn/news/16/). These data are from 50 to $170 \mathrm{~s}$ with an interval of $0.2 \mathrm{~s}$, corresponding to the process 


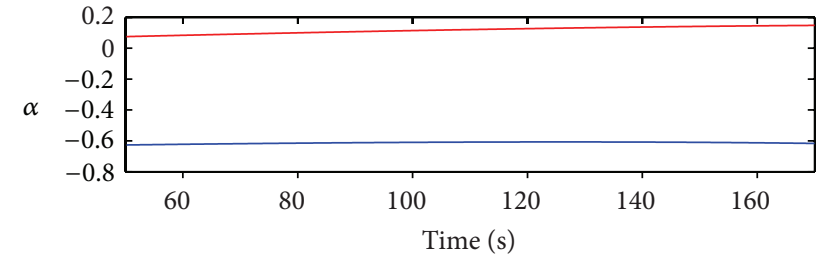

(a)

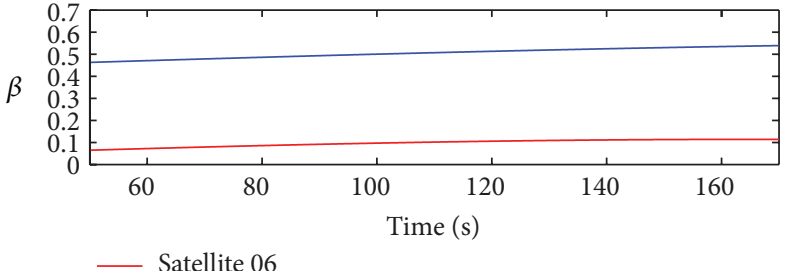

- Satellite 06

— Satellite 09

(b)

Figure 4: $\alpha$ and $\beta$ of observation satellites from $50 \mathrm{~s}$ to $170 \mathrm{~s}$.
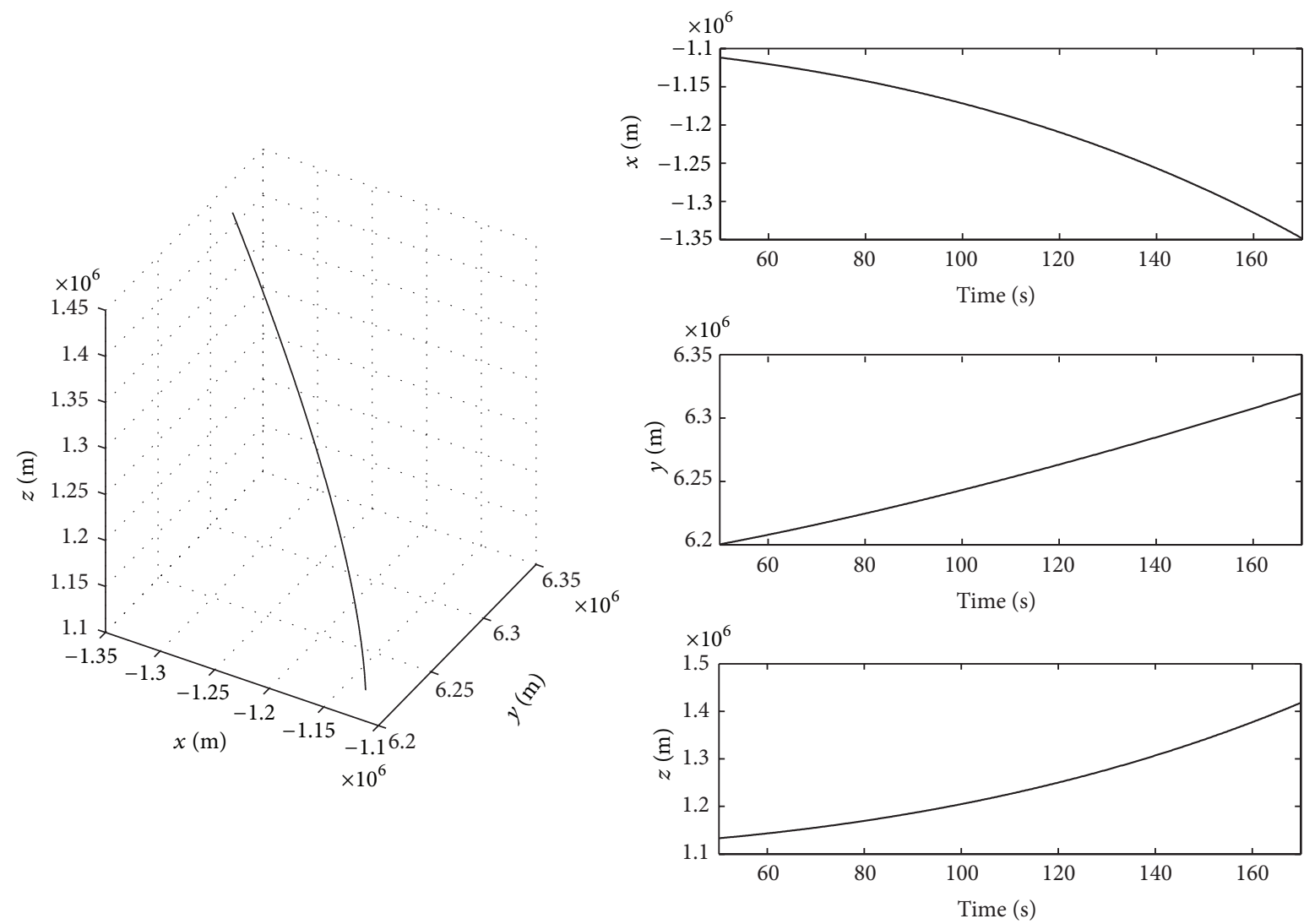

FiguRE 5: Trajectory (left) and instantaneous coordinates (right) of the aircraft.

of rocket propulsion. The trajectories of observation satellites are plotted in Figure 3. $\alpha$ and $\beta$ are plotted in Figure 4. Then the coordinates of aircraft in the fundamental coordinate system, which are used as observations, are determined according to formulas in Section 2 and are shown in Figure 5.

Four numerical experiments are designed to validate the sensitivity to the number of observations in the model. In experiment one (E1), the trajectory of aircraft is estimated by assimilating all observations. The number of observations is 601 in E1 with internal of $0.2 \mathrm{~s}$. In E2-E4, the number of observations is decreased successively, which is 61,21 , and 13 , respectively. In numerical experiments, the initial values of $F_{x}, F_{y}$, and $F_{z}$ are all equal to $10 \mathrm{~m}^{2} / \mathrm{s}$, and the time step is equal to $0.2 \mathrm{~s}$. Simulated results are illustrated as follows.
Simulated results show good agreement with the observed ones in all four experiments. However, they are not plotted in a figure for very small differences with each other and the observations. Table 1 lists the mean absolute differences (MADs) and mean relative differences (MRDs) between simulated results and observations. In all four experiments, the MADs and MRDs are all very small, indicating the reasonability and feasibility of the model. Log of cost function and gradient norm are plotted in Figure 6. Cost function and gradient norm decrease fast during the iteration, implying the efficiency of the typical steepest method. Careful inspection shows that the MAD and MRD, as well as the value of cost function at the end of iterations, increase from $\mathrm{E} 1$ to $\mathrm{E} 4$. This is to say, the trajectory estimation 


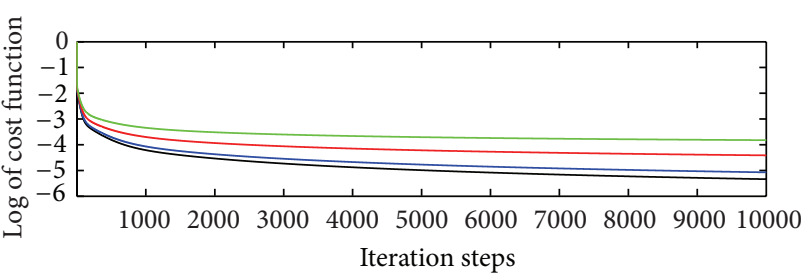

(a)

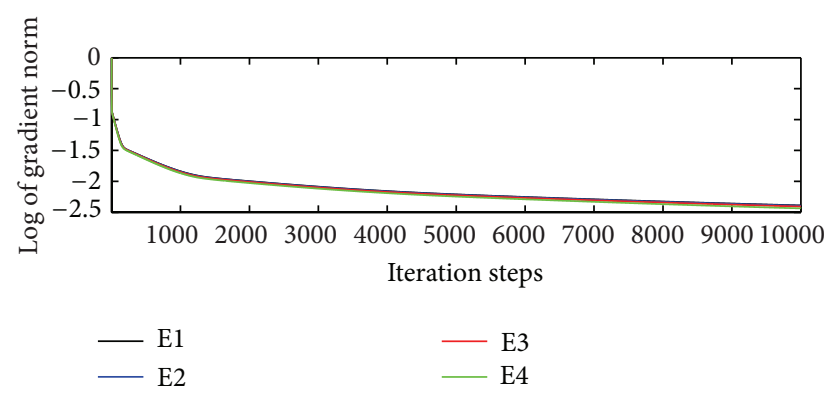

(b)

FIGURE 6: Log of cost function and gradient norm during iteration.

of the aircraft becomes worse as the number of observations is decreased. Combining all these results, we can draw two conclusions. One is that even by assimilating a small number of observations, the trajectory of aircraft can be estimated using the dynamic model with the adjoint method. The other is that the trajectory estimation can become more accurate by assimilating more observations.

\section{Summary and Conclusions}

In this paper, a double-satellite passive positioning system is constructed based on the theory of space geometry. In this system, two observation coordinate systems corresponding to two observation satellites and a fundamental coordinate system exist. In each observation coordinate system, there exists a ray from the observation satellite to the aircraft. In the ideal situation where no error exists in the double-satellite passive positioning system, these two rays intersect and the point of intersection is the aircraft. One difficulty lies in that these two rays may not intersect due to the existence of various errors. Under this situation, this work assumes that the middle point of common perpendicular between two rays is the actual position of aircraft. Based on the assumption and theory of space geometry, the coordinates of aircraft in the fundamental coordinate system can be determined.

In order to estimate the trajectory of aircraft during the process of rocket propulsion, a dynamic model with the adjoint method is constructed. The forward model is used to simulate the motion of aircraft, and the adjoint model is used to optimize parameters. By assimilating observations, which are the coordinates of aircraft in the fundamental coordinate system, the trajectory of aircraft can be estimated. Numerical experiments are designed to validate the reasonability and feasibility of this model. Results indicate that even by assimilating a small number of observations, the trajectory of aircraft can be estimated using the dynamic model with the adjoint method. In addition, the trajectory estimation can become more accurate by assimilating more observations.

\section{Acknowledgments}

Partial support for this research was provided by the National Natural Science Foundation of China through Grant 41206001 and 41076006, the Major State Basic Research Development Program of China through Grant 2013CB956500, the Natural Science Foundation of Jiangsu Province through Grant BK2012315, and the Priority Academic Program Development of Jiangsu Higher Education Institutions.

\section{References}

[1] K. Spingarn, "Passive position location estimation using the extended Kalman filter," IEEE Transactions on Aerospace and Electronic Systems, vol. 23, no. 4, pp. 558-567, 1987.

[2] R. Jamilnia and A. Naghash, "Simultaneous optimization of staging and trajectory of launch vehicles using two different approaches," Aerospace Science and Technology, vol. 23, pp. 8592, 2012.

[3] R. W. Lardner, "Optimal assimilation of current and surface elevation data in a two-dimensional numerical tidal model," Applied Mathematical Modelling, vol. 19, no. 1, pp. 30-38, 1995.

[4] J. Zhang and X. Lu, "Inversion of three-dimensional tidal currents in marginal seas by assimilating satellite altimetry," Computer Methods in Applied Mechanics and Engineering, vol. 199, no. 49-52, pp. 3125-3136, 2010.

[5] Z. Guo, A. Cao, and X. Lv, "Inverse estimation of open boundary conditions in the Bohai Sea," Mathematical Problems in Engineering, vol. 2012, Article ID 628061, 9 pages, 2012.

[6] A. Cao, Z. Guo, and X. Lv, "Inversion of two-dimensional tidal open boundary conditions of $\mathrm{M}_{2}$ constituent in the Bohai and Yellow Seas," Chinese Journal of Oceanology and Limnology, vol. 30, no. 5, pp. 868-875, 2012.

[7] H. Chen, C. Miao, and X. Lv, "A three-dimensional numerical internal tidal model involving adjoint method," International Journal for Numerical Methods in Fluids, vol. 69, no. 10, pp. 15841613, 2012.

[8] X. Lu and J. Zhang, "Numerical study on spatially varying bottom friction coefficient of a 2D tidal model with adjoint method," Continental Shelf Research, vol. 26, no. 16, pp. 1905-1923, 2006.

[9] J. Zhang, X. Lu, P. Wang, and Y. P. Wang, "Study on linear and nonlinear bottom friction parameterizations for regional tidal models using data assimilation," Continental Shelf Research, vol. 31, no. 6, pp. 555-573, 2011.

[10] L. Yu and J. J. O'Brien, "Variational estimation of the wind stress drag coefficient and the oceanic eddy viscosity profile," Journal of Physical Oceanography, vol. 21, pp. 709-719, 1990.

[11] H. Chen, C. Miao, and X. Lv, "Estimation of open boundary conditions for an internal tidal model with adjoint method: a comparative study on optimization Methods," Mathematical Problems in Engineering, vol. 2013, Article ID 802136, 12 pages, 2013. 


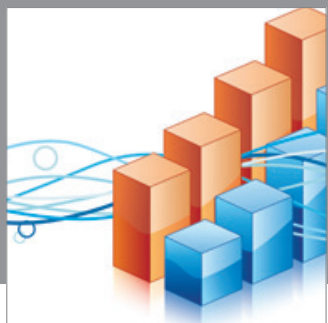

Advances in

Operations Research

mansans

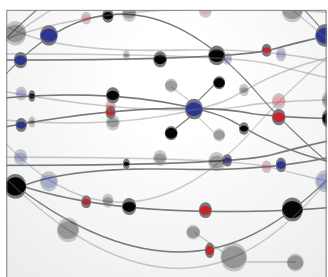

The Scientific World Journal
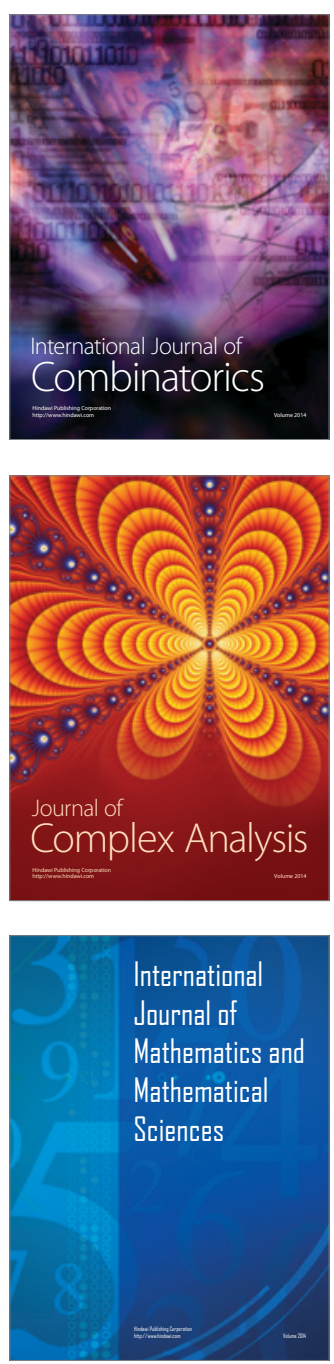
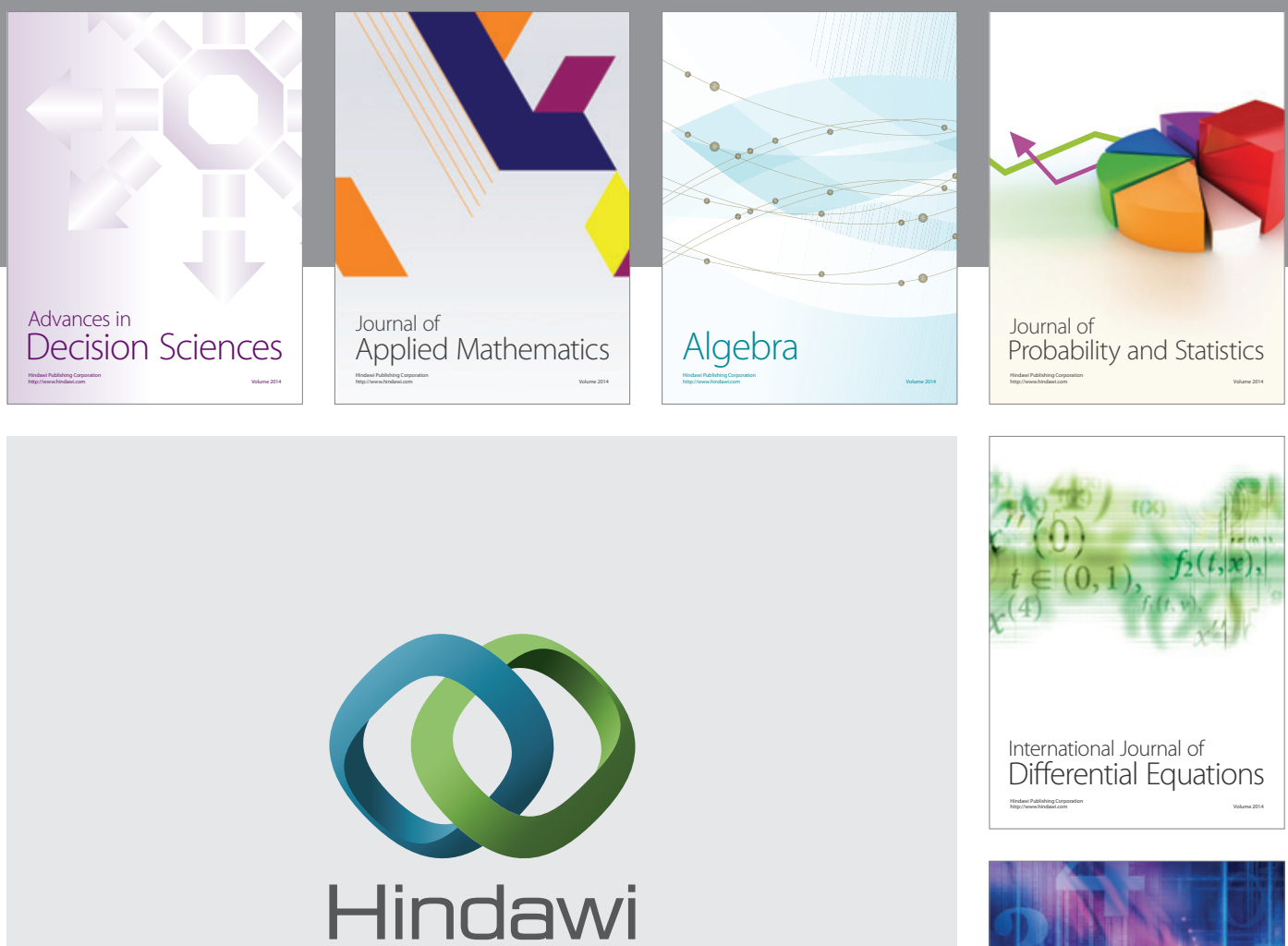

Submit your manuscripts at http://www.hindawi.com
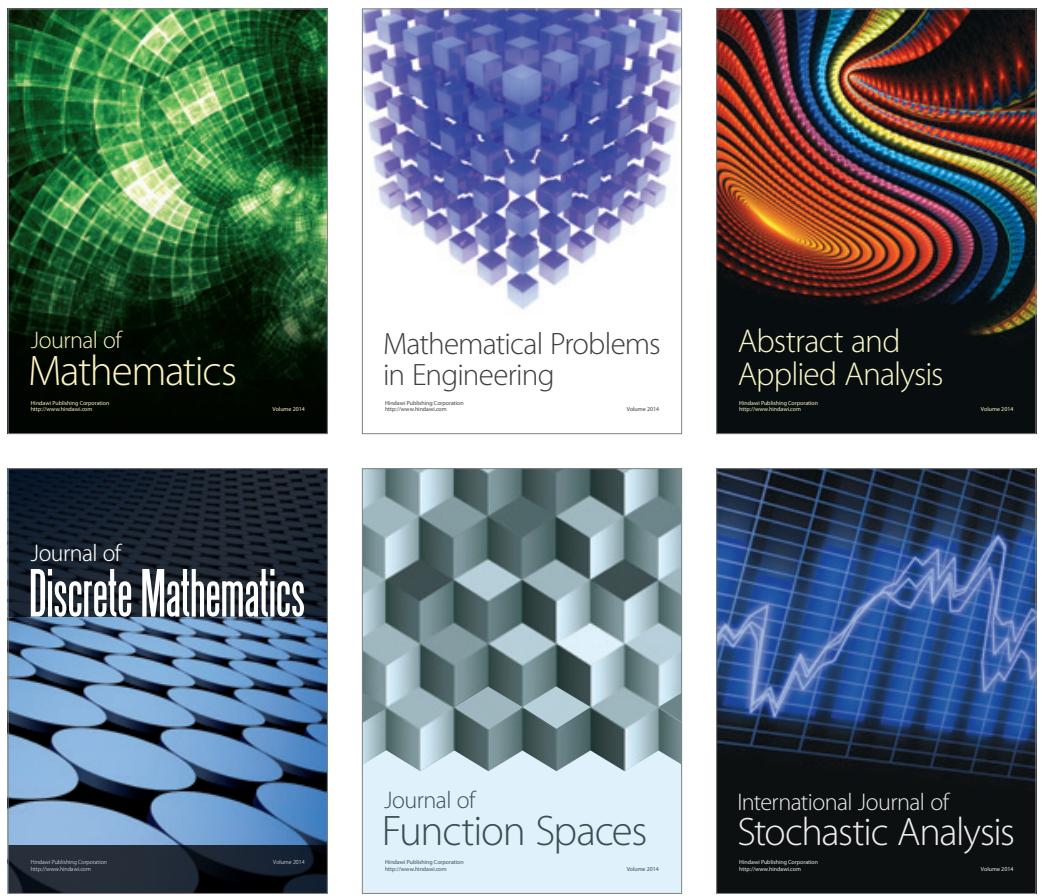

Journal of

Function Spaces

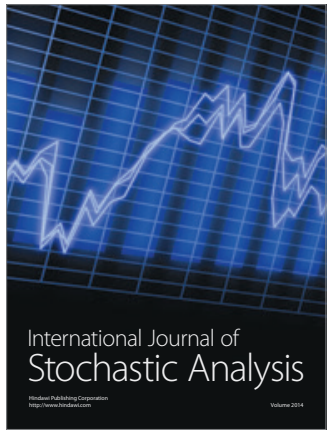

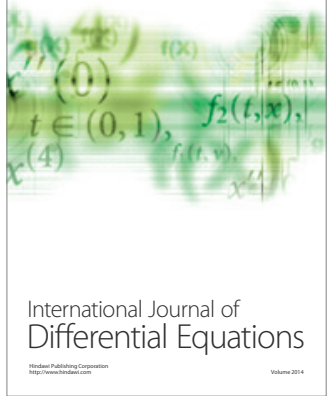
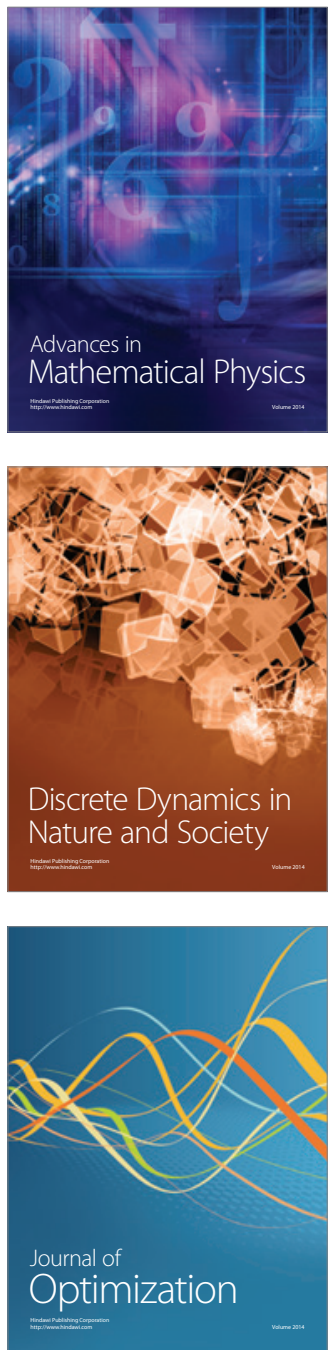